\title{
EFECTO BIOESTIMULANTE DE Trichoderma harzianum Rifai EN POSTURAS DE LEUCAENA, CEDRO Y SAMÁN
}

\section{Biostimulating effect of Trichoderma harzianum Rifai on seedling of Leucaena, Cedar and Saman}

\author{
Tarsicio Santana Díaz \& Leónides Castellanos González¹
}

Santana-Díaz, T., \&, Castellanos-González, L. (2018). Efecto bioestimulante de Trichoderma harzianum Rifai en posturas de Leucaena, Cedro y Samán. Colombia Forestal, 21(1), 81-90

Recepción: 25 de marzo de 2017

\section{Resumen}

El presente artículo evaluó el efecto bioestimulante de Trichoderma harzianum Rifai en la producción de postura de Leucaena leucocephala (Lam) de Wit., Cedrela odorata L. y Albizia saman (Jacq.) Merr. Se desarrollaron tres experimentos en condiciones de vivero, uno para cada especie. Se empleó un diseño completamente aleatorio con cuatro parcelas por tratamiento. En cada experimento los tratamientos fueron: Trichoderma harzianum a razón de 20 g.L ${ }^{-1}$, 40 g.L-1 y un testigo. Se evaluó el porcentaje de germinación y las variables morfométricas diámetro y altura del tallo, así como biomasa seca en raíz y parte aérea de la planta. Los tratamientos con Trichoderma no incrementaron el porcentaje de germinación en cedro, samán y leucaena. T. harzianum incrementó la altura, el número de hojas y la biomasa seca del área foliar en las plántulas de cedro, mientras que en leucaena y samán solo provocó incrementos del diámetro basal de las plántulas. Palabras clave: antagonista, biomasa, especies forestales, germinación.
Aprobación: 28 de agosto de 2017

\begin{abstract}
The present article aims to evaluate the biostimulating effect of Trichoderma harzianum Rifai in the seedling production of Leucaena leucocephala (Lam) de Wit., Cedrela odorata L., and Pithecellobium saman (Jacq.) Merr. An experiment for each specie was carried out under nursery conditions. A totally randomized design was used with four parcels by treatment. The treatments were: Trichoderma harzianum to reason of 20 g. $\mathrm{L}^{-1}$ and to 40 g.L $\mathrm{L}^{-1}$, and a control. The germination percentage was evaluated, and the morphometric variables diameter of the shaft, height and dry matter of the root and the air part of the plant. The treatments with Trichoderma didn't increase the germination percentage in cedar, saman and leucaena. T. harzianum increased the height, the number of leaves and the dry biomass of the foliar area in the cedar seedlings, while in leucaena and saman only it incremented the basal diameter of the seedlings.
\end{abstract}

Key words: antagonist, biomas, forestall species, germination

1 Facultad de Ciencias Agrarias. Universidad de Pamplona. Pamplona, Colombia. Iclcastell@gmail.com 


\section{INTRODUCCIÓN}

Los bioestimulantes son productos que contiene células vivas o latentes de cepas microbianas previamente seleccionadas que se caracterizan por producir sustancias fisiológicamente activas (auxinas, giberelinas, citoquininas, aminoácidos, péptidos y vitaminas). Cuando estas interactúan con la planta promueven diferentes eventos metabólicos en función de estimular su crecimiento y el desarrollo (Castillo et al., 2007).

Las especies del género Trichoderma son los antagonistas más utilizados para el control de enfermedades de plantas producidos por hongos debido a su inocuidad, a su facilidad para ser aisladas y cultivadas, así como a su crecimiento rápido en un gran número de sustratos (Valdés, 2014). En algunos países como México se recomienda aplicar de forma preventiva en los viveros forestales para la prevención de enfermedades radiculares ( $\mathrm{Ci}$ brián \& Cibrián, 2017). También se incluye entre los hongos bioestimulantes, ya que son capaces de promover el crecimiento y el vigor en las plantas por la habilidad que poseen para hacer que las raíces sean más robustas, logrando mayor profundidad, por lo que son más resistentes a las sequías y pueden absorber más nutrientes (Donoso et al., 2008).

Conociendo las características bioestimulantes de Trichoderma se han realizado diferentes investigaciones para verificar este efecto en plántulas de vivero de especies forestales y acortar el periodo en esta etapa (Donoso et al., 2008; Romero et al., 2008; Díaz \& Ayala, 2011; Ramírez et al., 2015).

Entre las especies forestales más cultivadas dentro del territorio del estado Apure, en Venezuela, se encuentran: Leucaena leucocephala (Lam.) de Wit. (leucaena), Cedrela odorata L. (cedro), Albizia saman (Jacq.) Merr. (samán), las cuales poseen alto valor económico, así como fácil adaptabilidad a los suelos (Inia, 2007).

L. leucocephala es una de las leguminosas originaria de América Central de uso múltiple más productiva y versátil disponible en regiones tropicales.
Se puede afirmar que es la más utilizada en sistemas extensivos de pastoreo y también para áreas de corte, además favorece el control de la erosión (Costa \& Durigan, 2010), con resultados satisfactorios en Venezuela (Clavero, 1998).

C. odorata es la especie del género de mayor importancia comercial y de mayor extensión. Por su aromática madera, posee una alta demanda en los trópicos americanos debido a que es naturalmente resistente a las termitas y a la pudrición (Betancourt, 2000).

El samán es un árbol muy importante en Latinoamérica como proveedor de sombra, especialmente en parques. En Venezuela está ampliamente distribuido en los Ilanos centrales. Sin embargo, su madera es muy demandada y lo han considerado bandera para la reforestación de algunas zonas de Aragua (Torres et al., 2011).

Por otra parte, Parra \& Gámez (2012) hacen referencia a que el cedro y el samán están en el Libro Rojo de la Flora de Venezuela como vulnerables, pasando de menor riesgo a casi amenazados. De ahí la importancia de lograr posturas de estas plantas con vigor y en el tiempo más corto posible en la etapa de vivero.

El objetivo del presente trabajo fue evaluar el efecto bioestimulante de Trichoderma harzianum en la producción de postura de los arboles forestales leucaena, cedro y samán.

\section{MATERIALES Y MÉTODOS}

La presente investigación se realizó en el vivero forestal Municipal ubicado en la población de Mantecal, municipio Muñoz, estado Apure, Venezuela, en el periodo comprendido de abril de 2012 a agosto de 2012. Se incluyeron las especies leucaena (L. leucocephala [Lam] de Wit), cedro (C. odorata L.) y samán (A. saman [Jacq.] Merr.).

El clima del municipio Muñoz está caracterizado por una temperatura media anual de $26.6^{\circ} \mathrm{C}$ y las precipitaciones estacionales oscilan entre 700 y $1500 \mathrm{~mm}$ anuales. Presenta dos estaciones bien 
diferenciadas: un período lluvioso desde mayo hasta octubre y otro seco, desde noviembre hasta abril. Su relieve está enmarcado dentro de la depresión central llanera, que apenas se levanta de 50 a 200 metros sobre el nivel del mar (Rodríguez et al., 2013).

El ensayo se desarrolló en bolsas de polietileno de $2 \mathrm{~kg}$, las cuales se llenaron con una mezcla de suelo del lugar y materia orgánica $(\mathrm{MO})$ de excretas de bovino descompuesta, en la proporción: $90 \%$ de suelo y $10 \%$ de $\mathrm{MO}$ (relación v/v).

El suelo empleado contó con las siguientes características: inceptisol franco limoso (relación limo/arcilla de 2.7), conductividad $\mathrm{ds} / \mathrm{m}=0.05, \mathrm{pH}$ en agua $=6.4 ; \mathrm{MO}(\%)=3.13 ; \mathrm{P}\left(\mathrm{mg} \mathrm{kg}^{-1}\right)=15.79$; $\mathrm{K}\left(\mathrm{mg} \mathrm{kg}^{-1}\right)=51.13$ y Ca $\left(\mathrm{mg} \mathrm{kg}^{-1}\right)=221.33$.

Como material de siembra se utilizaron semillas recolectadas en distintos sectores del municipio, las cuales garantizaron el 95 \% de germinación según prueba previa. Se depositaron dos semillas por bolsa. Se condujeron tres experimentos, uno para cada especie forestal basados en un diseño completamente al azar, con tres tratamientos consistentes en dos variantes con plaguicidas biológicos y un testigo sin tratamiento, con cuatro réplicas. Cada replica estuvo constituida por 20 bolsas.

Los tratamientos fueron los siguientes:

- Tratamiento con Trichoderma harzianum a razón de $20 \mathrm{~g} \mathrm{~L}^{-1}$.

- Tratamiento con Trichoderma harzianum a razón de $40 \mathrm{~g} \mathrm{~L}^{-1}$

Las dosis de ensayo se corresponden con las dosis máximas y mínimas recomendadas por Stefanova (2007) para el uso del biopreparado a base de Trichoderma, formulado sólido.

El bioproducto T. harzianum se obtuvo en el laboratorio de biocontroladores El Rescate Campesino, ubicado en el municipio de Biruaca del estado Apure, con una concentración entre $1-3 \times 10^{9} \mathrm{co}-$ nidios/g, sobre sustrato (arroz) con 25 días de cosechados, según especificaciones de calidad del producto.
En las dos variantes con T. harzianum se trataron las semillas por inmersión con este producto biológico a la dosis $20 \mathrm{~g} \mathrm{~L}^{-1}$ por un periodo de 10 minutos, posteriormente se secaron y sembraron.

En una de las variantes biológicas se aplicó $T$. harzianum a razón de $20 \mathrm{~g} \mathrm{~L}^{-1}$ cada 15 días después de la siembra con una solución final de 300 $\mathrm{L} \mathrm{ha}^{-1}$. En la otra variante biológica se aplicó T. harzianum a razón de $40 \mathrm{~g} \mathrm{~L}^{-1}$ cada 15 días después de la siembra con una solución final de $300 \mathrm{~L} \mathrm{ha}^{-1}$. Para la frecuencia de aplicación durante el ciclo de las plántulas se utilizó el criterio seguido por Bécquer et al. (2016) en los tratamientos al triticale Triticum aestivum $\mathrm{L}$.

En total, se realizaron seis tratamientos biológicos durante el ciclo de las posturas hasta los 75 días de edad.

Durante el período de desarrollo de las posturas fueron atendidas con respecto al riego, manejo y tratamientos insecticidas e insecticidas semanalmente, siguiendo las normas técnicas establecidas. Se realizaron deshierbes cada 15 días. Los riegos fueron diarios hasta un $80 \%$ de capacidad de campo con una regadera manual, los cuales fueron suspendidos en días posteriores a los que se produjeron lluvias.

Para determinar el efecto bioestimulador de antagonista se determinaron las siguientes variables: porcentaje de germinación después de la siembra 30 días (\%), altura (cm) de la planta a los 60 días, diámetro basal $(\mathrm{mm})$ a los 60 días y número de hojas a los 60 días.

Cuando el $50 \%$ al menos de las posturas alcanzó los requisitos establecidos para el trasplante (alrededor de los 90 días) se determinaron las siguientes variables: altura de la planta $(\mathrm{cm})$, diámetro basal $(\mathrm{mm})$, número de hojas y biomasa seca de la raíz (g) y de la parte aérea (g).

Estas variables han sido consideradas en estudios similares por otros investigadores como Medina et al. (2007) y Ramírez et al. (2015).

La altura de las plantas se midió con una regla graduada desde la base del cuello o tallo de la 
planta hasta la yema terminal y diámetro basal con un pie de rey a $5 \mathrm{~cm}$ de la base del tallo.

Para la determinación de las masas: parte aérea, radical, se utilizó el método gravimétrico (secado en estufa a $65^{\circ} \mathrm{C}$ hasta peso constante). Las plantas fueron cortadas en la base del cuello de la raíz. Se determinó por separado la biomasa seca de la raíz y de la parte aérea.

A la variable porcentaje de germinación se le realizó el análisis estadístico de comparación por la prueba de Kruskal-Wallis al comprobarse la no normalidad de los datos por la prueba de Kolmodorov-Smirnov. A la altura, número de hojas, diámetro basal de las posturas y biomasa seca en la parte aérea y las raíces se les aplicó Anova de un factor con el test de Tukey para $\mathrm{P} \leq 0.05$ utilizando el paquete estadístico SPSS (Statistical Package for the Social Sciences) para Windows versión 21.0 (IBM, 2012).

\section{RESULTADOS}

No se presentó efecto bioestimulador de los tratamientos realizados a la semilla y al suelo en relación con el porcentaje de germinación de las plantas de L. leucocephala, C. odorata y A. saman ya que los tratamientos con $T$. harzianum presentaron un $87.5 \%$ de germinación y en el testigo
$75 \%$ para la primera especie $(p=0.01)$, entre 95 y $87 \%$ (testigo, $p=0.01$ ) para la segunda y entre 100 y $91 \%$ (testigo, $p=0.01$ ) para la tercera, respectivamente.

Se manifestó un efecto bioestimulador ( $p=0.024)$ sobre las plantas de $L$. leucocephala al ser tratadas con T. harzianum, pues se observa mayor diámetro basal a los 60 días en las plantas que recibieron la dosis de la variante $T$. harzianum a $40 \mathrm{~g} \mathrm{~L}^{-1}$ seguidas por la que recibieron la dosis de T. harzianum al $20 \mathrm{~g} \mathrm{~L}^{-1}$, no difiriendo estadísticamente del tratamiento testigo. A los 90 días no se presentó diferencia estadística para esta variable de los tratamientos con relación al testigo (tabla 1).

Al analizar la variable altura de las plantas para esta especie se observaron valores entre $34 \mathrm{~cm} \mathrm{y}$ $45 \mathrm{~cm}$ al momento del trasplante, sin diferencia estadística en las evaluaciones a los 60 y 90 días respectivamente.

Los tratamientos biológicos con T. harzianum no indujeron un incremento significativo del número de hojas en la especie $L$. leucocephala en relación con el testigo. No se presentó aumento de la biomasa seca de las raíces y de la parte aérea de las plantas con la aplicación de T. harzianum en la especie L. leucocephala, con respecto al tratamiento testigo.

La variable diámetro basal varió en el ensayo C. odorata entre 4.8 y $5.3 \mathrm{~mm}$ a los 60 días

Tabla 1. Diámetro y altura de las plantas de L. leucocephala

\begin{tabular}{lcccc}
\hline \multicolumn{1}{c}{ Tratamientos } & $\begin{array}{c}\text { Diámetro a los 60 } \\
\text { días }\end{array}$ & $\begin{array}{c}\text { Diámetro a los 90 } \\
\text { días }\end{array}$ & $\begin{array}{c}\text { Altura a los 60 } \\
\text { días }\end{array}$ & $\begin{array}{c}\text { Altura a los 90 } \\
\text { días }\end{array}$ \\
\hline Unidad & $(\mathrm{mm})$ & $(\mathrm{mm})$ & $(\mathrm{cm})$ & $(\mathrm{cm})$ \\
T. harzianum a $20 \mathrm{~g} \mathrm{~L}^{-1}$ & $2.29 \mathrm{ab}$ & $4.32 \mathrm{NS}$ & $11.87 \mathrm{NS}$ & $34.72 \mathrm{NS}$ \\
T. harzianum a 40 g L-1 & $2.71 \mathrm{a}$ & $4.79 \mathrm{NS}$ & $11.64 \mathrm{NS}$ & $45.95 \mathrm{NS}$ \\
Testigo & $1.93 \mathrm{~b}$ & $3.93 \mathrm{NS}$ & $10.93 \mathrm{NS}$ & $34.23 \mathrm{NS}$ \\
Valor F (Fisher) del Anova & $4.25 *$ & $0.518 \mathrm{NS}$ & $2.41 \mathrm{NS}$ & $1.72 \mathrm{NS}$ \\
Error típico & 0.205 & 0.473 & 0.756 & 4.14 \\
Coeficiente de variación (\%) & 15.83 & 19.40 & 7.05 & 19.80 \\
\hline
\end{tabular}

Medias seguidas de letras distintas en las columnas difieren entre sí por el test de Tukey $(\mathrm{P}<0.05)$; **; * y NS: significativo (P<0.01); $(\mathrm{P}<0.05)$ y no significativo. Respectivamente. 
y entre 8.4 y $9.3 \mathrm{~mm}$ a los 90 días de edad, sin diferencia estadística entre las diferentes variantes estudiadas. Sin embargo, el tratamiento de T. harzianum a $40 \mathrm{~g} \mathrm{~L}^{-1}$ provocó un aumento de la altura de las plantas de C. odorata a los 60 días, ya que esta variante difiere estadísticamente $(p=0.005)$ del resto de los tratamientos, mostrando que hay un efecto bioestimulante sobre las plantas. En cuanto a la altura a los 90 días se observa que también la variante $T$. harzianum a $40 \mathrm{~g} \mathrm{~L}^{-1}$ difiere estadísticamente $(\mathrm{p}=0.007)$ del tratamiento testigo. (tabla 2).

A los 60 días de edad de las posturas de C. odorata se observó un incremento del número de hojas para los tratamientos con T. harzianum a 20 y $40 \mathrm{~g} \mathrm{~L}^{-1}$ con relación al testigo $(\mathrm{p}=0.004)$. Este efecto bioestimulante se observó también para esta especie de planta a los 90 días, el cual fue significativo $(p=0.006)$ para la variable $T$. harzianum a $40 \mathrm{~g} \mathrm{~L}^{-1}$ con relación al tratamiento testigo, lo cual no resultó así para T. harzianum a $20 \mathrm{~g} \mathrm{~L}^{-1}$. La aplicación del antagonista $T$. harzianum no provocó aumento de la biomasa de las raíces de las plántulas de C. odorata, pero sí sobre la biomasa seca $(p=0.008)$ en la parte aérea de la planta a las dos dosis estudiadas (tabla 3).

Esta situación resulta lógica si se tiene en cuenta que, como se ha evidenciado anteriormente, las posturas de C. odorata tratadas con T. harzianum presentaron incremento en altura a los 60 y 90 días y del número de hojas a los 60 y 90 días con respecto al tratamiento testigo.

En el caso del A. saman en la variable diámetro basal a los 60 días mostró incremento en los

Tabla 2. Diámetro y altura de las plantas de C. odorata

\begin{tabular}{lcccc}
\hline & $\begin{array}{c}\text { Diámetro a los 60 } \\
\text { días }\end{array}$ & $\begin{array}{c}\text { Diámetro a los 90 } \\
\text { días }\end{array}$ & $\begin{array}{c}\text { Altura a los 60 } \\
\text { días }\end{array}$ & $\begin{array}{c}\text { Altura a los 90 } \\
\text { días }\end{array}$ \\
\hline Tratamientos & $(\mathrm{mm})$ & $(\mathrm{mm})$ & $(\mathrm{cm})$ & $(\mathrm{cm})$ \\
T. harzianum a $20 \mathrm{~g} \mathrm{~L}^{-1}$ & $5.30 \mathrm{NS}$ & $9.22 \mathrm{NS}$ & $13.64 \mathrm{~b}$ & $59.25 \mathrm{ab}$ \\
T. harzianum a 40 g L-1 & $5.05 \mathrm{NS}$ & $9.25 \mathrm{NS}$ & $16.83 \mathrm{a}$ & $74.14 \mathrm{a}$ \\
Testigo & $4.82 \mathrm{NS}$ & $8.47 \mathrm{NS}$ & $12.41 \mathrm{~b}$ & $43.18 \mathrm{c}$ \\
Valor F (Fisher) del Anova & $0.538 \mathrm{NS}$ & $2.41 \mathrm{NS}$ & $14.30^{* *}$ & $12.13^{* *}$ \\
Error típico & 0.265 & 0.255 & 2.18 & 3.82 \\
Coeficiente de variación (\%) & 10.45 & 5.74 & 10.55 & 13.43 \\
\hline
\end{tabular}

Medias seguidas de letras distintas en las columnas difieren entre sí por el test de Tukey $(\mathrm{P}<0.05) ;{ }^{* *} ;{ }^{*}$ y NS: significativo (P<0.01); $(\mathrm{P}<0.05)$ y no significativo respectivamente.

Tabla 3. Número de hojas y biomasa de las plantas de C. odorata

\begin{tabular}{lcccc}
\hline & \multicolumn{2}{c}{ Número de hojas a los } & \multicolumn{2}{c}{ Biomasa } \\
\hline Tratamientos & 60 días & 90 días & Raíces $(\mathrm{g})$ & Parte aérea $(\mathrm{g})$ \\
T. harzianum a $20 \mathrm{~g} \mathrm{~L}^{-1}$ & $59.25 \mathrm{a}$ & $141.75 \mathrm{ab}$ & $3.79 \mathrm{NS}$ & $14.43 \mathrm{a}$ \\
T. harzianum a $40 \mathrm{~g} \mathrm{~L}^{-1}$ & $74.14 \mathrm{a}$ & $147.50 \mathrm{a}$ & $4.43 \mathrm{NS}$ & $16.28 \mathrm{a}$ \\
Testigo & $43.18 \mathrm{~b}$ & $134.50 \mathrm{~b}$ & $2.97 \mathrm{NS}$ & $4.82 \mathrm{~b}$ \\
Valor F (Fisher) del Anova & $12.13^{* *}$ & $6.72 * *$ & 2.30 & $20.18^{* *}$ \\
Error típico & 3.82 & 2.67 & 0.435 & 0.589 \\
Coeficiente de variación (\%) & 13.43 & 3.84 & 24.23 & 8.95 \\
\hline
\end{tabular}

Medias seguidas de letras distintas en las columnas difieren entre sí por el test de Tukey $(\mathrm{P}<0.05)$; **; * y NS: significativo (P<0.01); $(\mathrm{P}<0.05)$ y no significativo respectivamente. 
tratamientos con T. harzianum a $20 \mathrm{~g} \mathrm{~L}^{-1}$ y $T$. harzianum a $40 \mathrm{~g} \mathrm{~L}^{-1}$ con relación al testigo $(\mathrm{p}=0.021)$ (tabla 4)

A los 90 días de edad las posturas alcanzaban entre 6.38 y 7.07 mm de grosor, no evidenciándose diferencias entre los tratamientos en estudio, lo cual puede deberse a que al momento de la evaluación de las raíces se observaron numerosas modulaciones similares a las que desarrolla Rizhobium sp. en las leguminosas y que pudo estimular el crecimiento de las posturas en todas las variantes, incluyendo al testigo a desarrollarse esta simbiosis (tabla 5).

La variable altura de las posturas de samán varió entre 20.43 y $23.04 \mathrm{~cm}$ respectivamente a los
60 días y 33.5 y $45 \mathrm{~cm}$ a los 90 días, sin que se observaran diferencias entre las variantes estudiadas en ninguno de los dos momentos.

No se observó un incremento del número de hojas en los tratamientos con $T$. harzianum para las posturas de A. saman. A los 60 días se manifestaron entre 90 y 98 hojas por plantas, con un aumento considerable a los 90 días cuando alcanzaron entre 222 y 242 hojas por plantas. Tampoco se observó un incremento de la biomasa seca en las raíces y de la parte aérea de las plantas para las posturas de $A$. saman cuando fueron tratadas con T. harzianum en ninguna de las dosis en estudio.

Tabla 4. Diámetro y altura de las plantas del A. saman

\begin{tabular}{lcccc}
\hline \multicolumn{1}{c}{ Tratamientos } & Diámetro a & Diámetro a & \multicolumn{1}{c}{ Altura a los } & Altura a los \\
\hline & los 60 días & los 90 días & 60 días & 90 días \\
unidad & $(\mathrm{mm})$ & $(\mathrm{mm})$ & $(\mathrm{cm})$ & $(\mathrm{cm})$ \\
T. harzianum a $20 \mathrm{~g} \mathrm{~L}^{-1}$ & $4.31 \mathrm{a}$ & $6.67 \mathrm{NS}$ & $22.39 \mathrm{NS}$ & $44.45 \mathrm{NS}$ \\
T. harzianum a $40 \mathrm{~g} \mathrm{~L}^{-1}$ & $4.31 \mathrm{a}$ & $7.07 \mathrm{NS}$ & $23.04 \mathrm{NS}$ & $46.27 \mathrm{NS}$ \\
Testigo & $3.29 \mathrm{~b}$ & $6.58 \mathrm{NS}$ & $20.43 \mathrm{NS}$ & $33.50 \mathrm{NS}$ \\
Valor F (Fisher) del Anova & $5.83 *$ & $0.73 \mathrm{NS}$ & $1.62 \mathrm{NS}$ & $2.64 \mathrm{NS}$ \\
Error típico & 0.20 & 0.422 & 0.87 & 2.16 \\
Coeficiente de variación (\%) & 3.97 & 12.65 & 16.45 & 9.66 \\
\hline
\end{tabular}

Medias seguidas de letras distintas en las columnas difieren entre sí por el test de Tukey $(\mathrm{P}<0.05) ;{ }^{* *} ;{ }^{*}$ y NS: significativo (P<0.01); $(\mathrm{P}<0.05)$ y no significativo respectivamente.

Tabla 5. Número de hojas y biomasa de las plantas de $A$. saman

\begin{tabular}{lcccc}
\hline & \multicolumn{2}{c}{ Número de hojas a los } & \multicolumn{2}{c}{ Biomasa } \\
\hline Tratamientos & 60 días & 90 días & Raíces $(\mathrm{g})$ & Parte aérea $(\mathrm{g})$ \\
T. harzianum a $20 \mathrm{~g} \mathrm{~L}^{-1}$ & $96.66 \mathrm{NS}$ & $222.39 \mathrm{NS}$ & $3.82 \mathrm{NS}$ & $10.94 \mathrm{NS}$ \\
T. harzianum a $40 \mathrm{~g} \mathrm{~L}^{-1}$ & $97.58 \mathrm{NS}$ & $234.20 \mathrm{NS}$ & $3.62 \mathrm{NS}$ & $10.71 \mathrm{NS}$ \\
Testigo & $90.79 \mathrm{NS}$ & $222.78 \mathrm{NS}$ & $2.62 \mathrm{NS}$ & $7.98 \mathrm{NS}$ \\
Valor F (Fisher) del Anova & $0.106 \mathrm{NS}$ & $0.971 \mathrm{NS}$ & $0.674 \mathrm{NS}$ & $0.940 \mathrm{NS}$ \\
Error típico & 9.77 & 9.75 & 0.798 & 1.40 \\
Coeficiente de variación (\%) & 20.43 & 8.45 & 34.95 & 28.18 \\
\hline
\end{tabular}

NS: Medias en las columnas no difieren entre sí por la prueba de Tukey $(\mathrm{P}<0.05)$. 


\section{DISCUSIÓN}

Los resultados obtenidos relacionados con la estimulación de la germinación de la semilla concuerdan con los de Díaz \& Ayala (2011) cuando trataron solamente con $T$. harzianum las semillas de majagua (Talipariti elatum [Sw.] Fryxell), pero no con los de Ramírez et al. (2015), quienes observaron incrementos de porcentajes de germinación en otras especies forestales soplillo (Lysiloma behanensis L.), yarúa (Caesalpinia violacea, [Mill.] Standl) y albizzia (Albizia procera [Roxb.] Benth) al aplicar T. harzianum con relación los testigos, pero para estas especies los testigos sin tratamiento del antagonista presentaron porcentajes de germinación entre 63 y $75 \%$.

Los valores de diámetro basal obtenidos para leucaena se corresponden con los obtenidos por otros investigadores en las condiciones de Cuba (Hernández \& Seguí, 1997), mientras que los valores de altura obtenidos para leucaena se corresponden con los informados por otros investigadores en las condiciones del estado de Aragua en Venezuela al momento del trasplante, los cuales fueron alrededor de $45 \mathrm{~cm}$ (Medina et al., 2007).

El número de hojas por plantas obtenidas fue superior a 1500 en todos los tratamientos, lo cual se corresponde a los observado por otros investigadores (Medina et al., 2007), quienes informaron más de 1000 hojas por planta, lo cual da una idea del desarrollo que alcanzaron las posturas de leucaena. El no incremento de la biomasa con los tratamientos biológicos resulta lógico si se tiene en cuenta que el biopreparado no tuvo un efecto sobre las plantas en las variables, altura, diámetro basal a los 90 días ni número de hojas.

No se encontraron trabajos donde se haya aplicado T. harzianum en la obtención de posturas de L. leucocephala, aunque se conoce que no todas las especies de plantas responden de igual forma a los metabolitos bioestimulantes que produce $T$. harzianum; y al parecer por ser L. leucocephala una especie bien adaptada a variadas condiciones edafológicas y ambientales, este efecto no se hizo evidente. La alta adaptabilidad de L. leucocephala se sustenta por su calidad nutricional, fijación de nitrógeno, crecimiento, tolerancia a la sequía y adaptación al ramoneo es la especie utilizada con mayor éxito en sistemas silvopastoriles intensivos en las regiones tropicales y subtropicales como ha sido señalado para Venezuela (Clavero, 1998). Al respecto Melo-Silva et al. (2014), a partir de una investigación realizada en Brasil, plantean que esta especie reúne todos los atributos para ser considerada una planta invasora.

El establecimiento de leucaena en los sistemas de producción agropecuarios tropicales mejora las condiciones químicas de los suelos, constituyéndose una alternativa en suelos deficientes en nutrientes (Razz \& Clavero, 2006). Además, Urbano et al. (2002) mencionan trabajos con leucaena en el sur del lago de Maracaibo, Venezuela, y destacan muy buenos resultados en las asociaciones con gramíneas.

No se encontró en la literatura informes del efecto biorregulador de Trichoderma sobre C. odorata. Sin embargo, sobre esta planta en la presente investigación en los tratamientos con este bioproducto se incrementaron las variables altura, número de hojas a los 60 y 90 días y biomasa seca de la parte aérea de la planta, por lo que esta podría ser una alternativa viable para el cedro, lo cual debe validarse en mayores extensiones en diferentes condiciones agroclimáticas.

El rápido desarrollo de samán se atribuye a que, como se conoce, esta es una planta que se desarrolla fácilmente bajo las condiciones de los llanos venezolanos, manifestando así una gran adaptabilidad en estas condiciones, lo cual se evidencia con el diámetro basal y la altura alcanzados, lo que también es apoyado por lo que se ha planteado con relación a la adaptabilidad de esta planta en la zona de Mantecal por Inia (2007) y a lo que contribuyó la presencia en las raíces de nódulos de Rizhobium sp.

Esto evidencia que se produjo un desarrollo acelerado de las posturas entre los 60 y 90 días, lo que se atribuye la gran adaptabilidad de la especie 
a las condiciones del ensayo. Con relación a la gran adaptabilidad del samán, Trujillo (2009) señala que se establece a una altitud: entre 0 a 1300 msnm bajo un clima con temperatura media de 15 a $22^{\circ} \mathrm{C}$, Iluvia anual de 600 a $3000 \mathrm{~mm}$, es resistente a condiciones de sequía y exigente al demandar luz.

El no incremento de biomasa seca en samán es lógico si se tiene en cuenta que la única variable que se incrementó con el biopreparado fue el diámetro basal y solo a los 60 días de edad, ya que a los 90 días este efecto bioestimulador no era evidente. En otras investigaciones realizadas con el samán en Venezuela no hubo incremento del número de hojas y la altura de la planta, a pesar de haberse incrementado el porcentaje de germinación en algunos pretratamientos a la semilla (Ramírez et al., 2013), lo que demuestra la alta capacidad de adaptación de esta planta forestal en estos ecosistemas.

Al analizar en su conjunto el efecto bioestimulador de las tres especies puede observarse que el tratamiento con T. harzianum provocó incrementos del diámetro basal a los 60 días para leucaena y samán, mientras que estimuló la altura a los 60 días y 90 días, el número de hojas a los 60 y 90 días y la materia seca del follaje para el cedro.

Para leucaena y samán se presentó un comportamiento similar con respecto al efecto de la bioestimulación (solo en el diámetro basal a los 60 días y no a los 90 días), aunque no se ha encontrado en la literatura información relacionada a esta situación, hay que tener en cuenta lo planteado con respecto a la rusticidad y adaptabilidad de leucauena y samán y además por ser leguminosas (familia Fabaceae), que tienen la posibilidad de fijar $\mathrm{N}$ atmosférico y entonces producirse a partir de la simbiosis de la planta con las bacterias nitrificantes naturales en el suelo, un desarrollo no controlado dentro de las variantes del experimento, lo cual no se produce en el cedro.

Al respecto efectos positivos, se informan en la literatura para otros forestales de la familia Fabaceae. Ramírez et al. (2015) verificaron un aumento del diámetro basal con el empleo de Trichoderma en las tres plantas forestales que estudiaron de esta familia: yarúa, soplillo y albizia, mientras que Romero et al. (2008) observaron que T. harzianum tenía efecto promotor del crecimiento en plántulas de tres forestales de otras familias; Eucalyptus grandis W. Hill. ex Maiden, Eucalyptus globulus Labill. (familia Myrtaceae) y Pinus taeda L. (familia Pinaceae); al igual que Danoso et al. (2008), quienes informaron incremento del desarrollo de Pinus radiata D. (familia Pinaceae) al aplicar una cepa de este antagonista en condiciones de vivero.

\section{CONCLUSIONES}

Los tratamientos con Trichoderma no incrementaron el porcentaje de germinación en cedro, samán y leucaena.

T. harzianum incrementó la altura, el número de hojas y la biomasa seca del área foliar en las plántulas de cedro, mientras que en leucaena y samán solo provocó incrementos del diámetro basal de las plántulas a los 60 días bajo las condiciones de los Ilanos de Apure en Venezuela.

\section{CONFLICTO DE INTERESES}

El autor declara no tener conflicto de intereses.

\section{CONTRIBUCIÓN POR AUTOR}

El autor único es responsable de la obra en todos los aspectos que condujeron a la elaboración de su publicación.

\section{REFERENCIAS BIBLIOGRÁFICAS}

\author{
Bécquer C.J., Puentes A.B., Ávila U., Quintana M., Yal- \\ dreisy G.Y., Medinilla F., \& Mirabales A. (1916). \\ Efecto de la inoculación con Bradyrhizobium sp. y
}


Trichoderma harzianum en triticale (X. Triticosecale Wittmack), en condiciones de estrés por sequía. Pastos y Forrajes, 39(1), 19-26.

Betancourt, A. (2000). Árboles maderables exóticos en Cuba. La Habana: Editorial Científico Técnica. 352 p.

Castillo, G., Gregorí, B.S., Michelena, G., Díaz de ViIlegas, M.E.; Delgado, G., Montano, R., Cejas, G., \& Gálvez, L.O. (2007). Bioproductos para la agricultura: surgimiento y desarrollo en el ICIDCA. Sobre los derivados de la caña de azúcar. Revista ICIDCA, 41(3), 42-51.

Cibrián, D., \& Cibrián, J. (2017). Estado del arte de la línea de investigación de plagas y enfermedades de importancia en viveros forestales. Texcoco, México: Red Temática en Salud Forestal.

Costa, J.N.M.N., \& Durigan, G. (2010). L. leucocephala (Lam.) de Wit (Fabaceae): Invasora ou ruderal? Revista Árvore, 34(5), 825-833.DOI: http://dx.doi. org/10.1590/S0100-67622010000500008

Clavero, T. (1998). Leucaena leucocephala. Alternativa para la alimentación animal. Caracas: Fundación Polar, Universidad del Zulia-Centro de Transferencia de Tecnología en Pastos y Forrajes. 78 p.

Díaz, M.C., \& Ayala, J.L. (2011). Influencia de Trichoderma harzianum a-34 en la germinación de las semillas de majagua (Talipariti elatum (Sw.) Fryxell). Fitosanidad, 15(3), 159-161.

Donoso, E., Lobos, G., \& Rojas, N. (2008). Efecto de Trichoderma harzianum y compost sobre el crecimiento de plántulas de Pinus radiata en vivero. Bosque, 29(1), 52-57. https://doi.org/10.4067/ S0717-92002008000100006

Hernández L., \& Segui E. (1997). Comportamiento de Leucaena spp en fase de vivero. Pastos y Forrajes, 20, 21-27.

International Business Machines (IBM). (2012). IBM SPSS Statistics for Windows. Version 21.0. New York: IBM Corp. Recuperado de: www.ibm.com/ analytics/us/en/technology/spss/

Instituto Nacional de Investigaciones Agrícolas (Inia). (2007). Caracterización socioeconómica del sector agrícola, Eje Norte Ilanero, Estado Apure. Maracay:
Informe Técnico de Resultados del Subproyecto. Primera Versión. 53 p.

Medina, M.C., Garcy, D.E., \& Iglesias J.M. (2007). Estudio comparativo de Moringa oleifera y Leucaena leucocephala durante la germinación y la etapa inicial de crecimiento. Zootecnia Tropical, 25(2), 83-93.

Melo-Silva, C., Peres, M.P., Mesquita, J.E., Bastos, B., \& Gonçalves-Barbosa, I.A. (2014). Biologia reprodutiva de L. leucocephala (Lam.) R. de Wit (Fabaceae: Mimosoideae): sucesso de uma espécie invasora. Neotropical Biology and Conservation, 9(2), 91-97. DOI: https://doi.org/10.4013/nbc.2014.92.03

Parra J., \& Gámez L. (2012). Determinación de especies arbóreas a través de caracteres vegetativos en la Estación Experimental Caparo, estado Barinas, Venezuela. Revista Forestal Venezolana, 56(2), 135-145.

Ramírez, M., Mesa. J.R., \& Soto R. (2015). Alternativas agroecológicas en la producción de posturas de tres especies forestales en el municipio de Aguada de Pasajeros. Agroecosistemas, 3(1), 387-400.

Ramírez, M., Urdaneta, A., Brigida Caraballo, B., \& García, D.E. (2013). Emergencia y desarrollo inicial de cuatro leguminosas forrajeras arbóreas presentes en la altiplanicie de Maracaibo, Venezuela. Pastos y Forrajes. 36(3), 303-312.

Razz, R., \& T. Clavero. (2006). Cambios en las características químicas de suelos en un banco Leucaena leucocephala y en un mono cultivo de Brachiaria brizantha. Revista de la Facultad de Agronomía de la Universidad del Zulia, 23(3), 331-337.

Romero, G., Crosara, A., \& Baraibar A. (2008). Trichoderma harzianum un biocontrol y biopromotor en vivero de especies forestales. Ciencia e Investigación Forestal. Instituto Forestal, 14(2), 335-345.

Ruiz, T. E., \& Febles, G. (1987). Leucaena (Leucaena leucocephala (Lam.) de Wit.) En: Una opción para la alimentación bovina en el trópico y subtrópico. La Habana: Editorial Edica. $200 \mathrm{p}$

Rodríguez, A., Castellanos, L., Sevilla, J., Aro, V., \& Peña, M. (2013). Caracterización del agroecosistema de la parroquia Rincón Hondo del municipio Muñoz, estado Apure. Agroecosistemas, 1(2), 123-134. 
Stefanova, M.M. (2007). Control de enfermedades fúngicas del suelo con biopreparados de Trichoderma harzianum. Curso internacional de sanidad vegetal. MINAG. pp.1-8.

Torres A., Vilanova, E., Ramírez H., Ninín B., Mora B., \& Hernández D. (2011). Una propuesta para el desarrollo forestal a pequeña escala en el sur del estado Aragua, Venezuela. Revista Forestal Venezolana, 55(1), 47-60.

Trujillo E. (2009). Guía de reforestación. Segunda edición, ilustrada, aumentada y actualizada. Bogotá: Editorial Dayber. 255 p.
Urbano, D., Dávila, C., Saavedra S., Rivas N., \& Morantes W. (2002). Impacto económico sobre la producción de leche en un sistema de pastoreo con asociaciones gramíneas leguminosas arbóreas en el sur del lago de Maracaibo. Venezuela. Archivo Latinoamericano de Producción Animal, 10(2), 102-116.

Valdés E. L. (2014). Caracteres principales, ventajas y beneficios agrícolas que aporta el uso de Trichoderma como control biológico. Agroecosistemas, 2(1), 254-264. 